\title{
Françoise Morin
}

Anthropologue, professeure émérite, Université Lumière, Lyon 2

professeure associée, dép. d'anthropologie, Université Laval et chercheure associée, CIERA.

(1975)

\section{"Roger Bastide ou l'anthropologie des gouffres."}

Un document produit en version numérique par Jean-Marie Tremblay, bénévole, professeur de sociologie au Cégep de Chicoutimi

Courriel: jean-marie tremblay@uqac.ca

Site web pédagogique : http://www.uqac.ca/jmt-sociologue/

Dans le cadre de la collection: "Les classiques des sciences sociales"

Site web: http://www.uqac.ca/Classiques_des_sciences_sociales/

Une collection développée en collaboration avec la Bibliothèque

Paul-Émile-Boulet de l'Université du Québec à Chicoutimi

Site web: http://bibliotheque.uqac.ca/ 


\section{Politique d'utilisation de la bibliothèque des Classiques}

Toute reproduction et rediffusion de nos fichiers est interdite, même avec la mention de leur provenance, sans l'autorisation formelle, écrite, du fondateur des Classiques des sciences sociales, Jean-Marie Tremblay, sociologue.

Les fichiers des Classiques des sciences sociales ne peuvent sans autorisation formelle:

- être hébergés (en fichier ou page web, en totalité ou en partie) sur un serveur autre que celui des Classiques.

- servir de base de travail à un autre fichier modifié ensuite par tout autre moyen (couleur, police, mise en page, extraits, support, etc...),

Les fichiers (.html, .doc, .pdf., .rtf, .jpg, .gif) disponibles sur le site Les Classiques des sciences sociales sont la propriété des Classiques des sciences sociales, un organisme à but non lucratif composé exclusivement de bénévoles.

Ils sont disponibles pour une utilisation intellectuelle et personnelle et, en aucun cas, commerciale. Toute utilisation à des fins commerciales des fichiers sur ce site est strictement interdite et toute rediffusion est également strictement interdite.

L'accès à notre travail est libre et gratuit à tous les utilisateurs. C'est notre mission.

Jean-Marie Tremblay, sociologue

Fondateur et Président-directeur général, LES CLASSIQUES DES SCIENCES SOCIALES. 
Cette édition électronique a été réalisée par Jean-Marie Tremblay, bénévole, professeur de sociologie au Cégep de Chicoutimi à partir de l'article de :

\section{Françoise Morin}

Université Lumière, Lyon 2

\section{"Roger Bastide ou l'anthropologie des gouffres."}

Un article publié dans la revue Archives des sciences sociales des religions, no 40, juillet-décembre 1975, pp. 99-106. Paris : CNRS.

[Le 3 mars 2008, Mme Françoise Morin nous donnait sa permission de diffuser cet article dans Les Classiques des sciences sociales.]

Courriel : $\quad$ Francoise.Morin@univ-lyon2.fr

Polices de caractères utilisée :

Pour le texte: Times New Roman, 14 points.

Pour les citations : Times New Roman 12 points.

Pour les notes de bas de page : Times, New Roman 10 points.

Édition électronique réalisée avec le traitement de textes Microsoft Word 2004 pour Macintosh.

Mise en page sur papier format : LETTRE (US letter), 8.5’’ x 11’')

Édition complétée le 16 juin 2008 à Chicoutimi, Ville de Saguenay, province de Québec.

\section{Fait avec}




\section{Françoise Morin}

Anthropologue, professeure émérite, Université Lumière, Lyon 2

professeure associée, dép. d'anthropologie, Université Laval et chercheure associée, CIERA.

\section{"Roger Bastide ou l'anthropologie des gouffres.”}

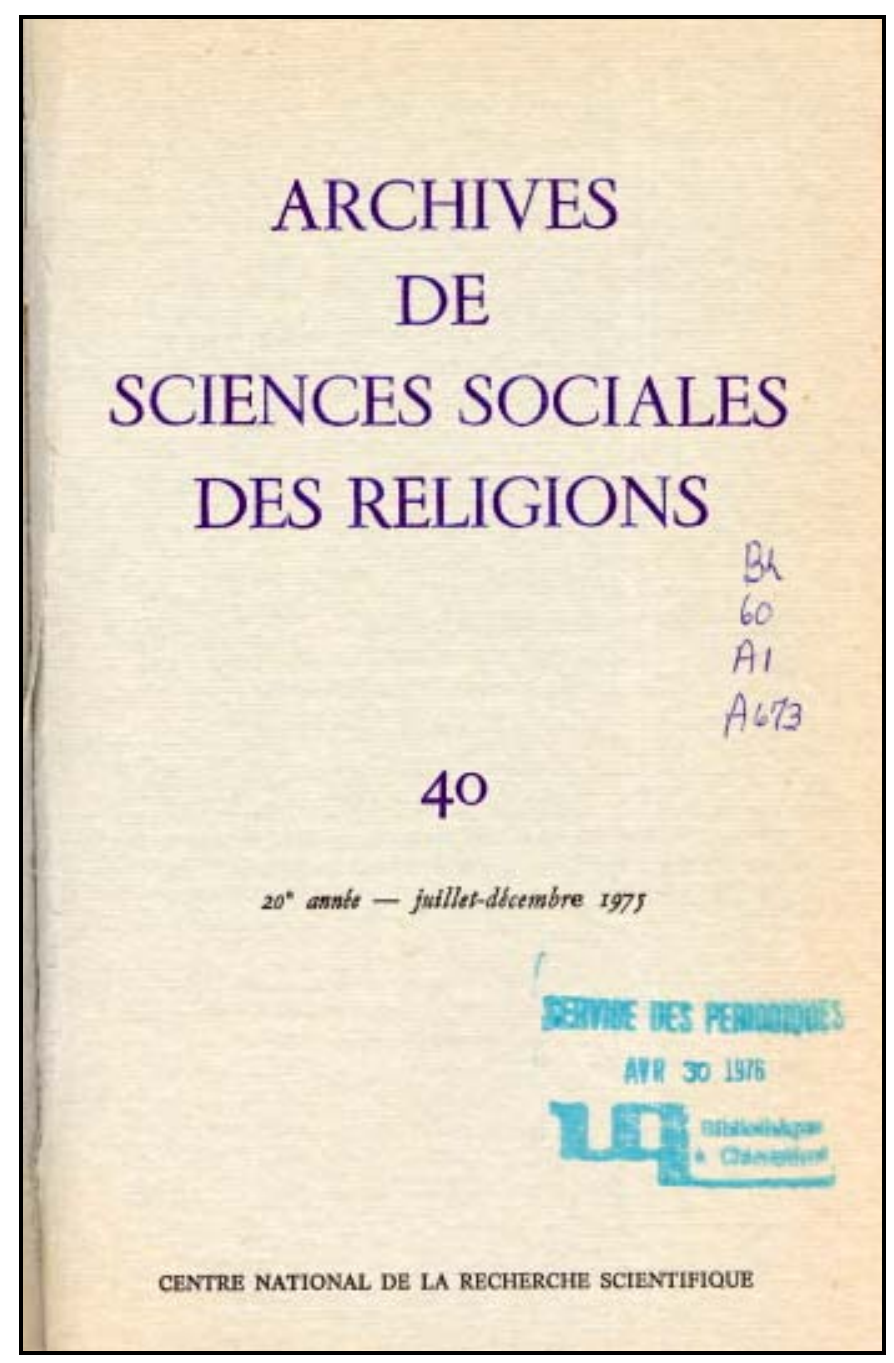

Un article publié dans la revue Archives des sciences sociales des religions, no 40, juillet-décembre 1975, pp. 99-106. Paris : CNRS. 


\section{Françoise Morin}

Anthropologue, professeure émérite, Université Lumière, Lyon 2

professeure associée, dép. d'anthropologie, Université Laval et chercheure associée, CIERA.

“Roger Bastide ou l'anthropologie des gouffres”. *

Un article publié dans la revue Archives des sciences sociales des religions, no 40, juillet-décembre 1975, pp. 99-106. Paris : CNRS.

In an article written in $1959 \mathrm{R}$. Bastide said that the anthropologist who hears the call of primitive civilizations is as it " were charmed by the abysses ». For R. B. himself this call took the shape of a mystic search and led him in 1938 to Latin America where for sixteen years he applied himself to apprehending the Other, listening and attempting to be accepted ; to do this he went so far as to share the Other's existential experience of the Sacred.

Upon his return to France, R. B. treated this « empiric »material theoretically and elaborated a new religious Anthropology around two major poles ; I) religions are to be considered as systems of symbols which must be apprehended in their living unity, as a total cultural activity ; 2) religion is to be treated like any other social fact, as something that changes while it continues resisting, and resists while it continues changing.

Choisit-on l'objet de ses études ou, aussi paradoxal que cela puisse paraître, est-ce l'objet qui vient vous chercher, s'accroche à vous, s'impose à votre réflexion ? ».

Cette question que se pose Roger Bastide au début de l'Introduction, encore inédite, à ses recherches sur \#Les Hommes de Couleur dans une Société blanche », et dont il décrit l'itinéraire géographique dans un premier chapitre intitulé « De Bahia, ville de tous les Saints, au Quartier Latin de Paris via São Paulo », cette question est pour lui capitale. Il nous en donne d'ailleurs les raisons. Dans le cas où l'on

* Texte présenté au Colloque de Cérisy-la-Salle, « Du religieux aujourd'hui » (20-30 août 1975), organisé par M. de Certeau, H. Desroche et H. Gouhier. 
choisit son objet de recherche « on le choisit, écrit-il, pour des raisons qui s'enracinent dans votre subjectivité et peuvent plonger parfois jusqu'aux structures de l'inconscient ». Bastide voit dans le choix de cet objet un reflet de la personnalité du chercheur et la construction qui s'élabore par la suite et qui dérive de l'observation est un mixte, selon lui, du sujet et de l'objet. Bastide n'était pas l'homme des « confessions ». Il parlait fort peu de lui-même et son œuvre ne foisonne pas de références personnelles. Nous avons cependant en filigrane dans ce texte écrit deux ans avant sa mort une des clefs de son anthropologie religieuse - l'union intime du sujet et de l'objet. Dans le cas contraire, où l'objet de recherche vous choisit, « il intervient, écrit Bastide, à un montent précis d'un long itinéraire intellectuel qui ne le visait pas à son point de départ ». C'est la rencontre de cet objet sur le chemin bastidien (lui explique l'étude des relations raciales au Brésil, alors que la préoccupation première était «la connaissance des survivances religieuses des esclaves noirs ».

Cette auto-critique que R. Bastide apporte à sa problématique est pour nous fondamentale, car elle nous indique très clairement que l'objet choisi, l'objet premier de ce chercheur fut le religieux, l'expérience existentielle du sacré, le mysticisme, la sociologie des gouffres... et que cet objet peut, en quelque sorte, nous servir de Rorschach pour appréhender sa personnalité.

Ayant eu le très grand bonheur pendant douze ans, au sein du Centre de Psychiatrie Sociale que Roger Bastide dirigea de 1962 à 1974, de participer à la fin de cet itinéraire intellectuel non " programmé au départ », noirs serions certes plus à l'aise en évoquant le « sociologue total », comme le décrit Jean Carbonnier dans L'Année Sociologique 1, ou le théoricien de la sociologie des rencontres de civilisations, ou le pionnier en recherches interdisciplinaires, ou bien le sociologue des maladies mentales. Car tenter de présenter l'anthropologue religieux C'est avant tout, comme il nous le fait remarquer lui-même, réfléchir

1 3e série, vol. 24, 1973, p. 6. 
sur l'homme et sa personnalité. Nous regrettons avec Henri Desroche ${ }^{2}$ que Roger Bastide n'ait pu accomplir « en ce dernier automne » ce qu'il avait accepté de nous livrer -son itinéraire en méthodologie religieuse -, lequel nous aurait permis de mieux comprendre l'implication de l'homme dans l'oeuvre de l'anthropologue. Ses dernières notes manuscrites témoignent, sous forme d'aide-mémoire, de ce souci : «Essayer de cerner les conditions de notre subjectivité. L'étude du sacré étant la rencontre de plusieurs expériences auxquelles sont intimement mêlées les nôtres »... Quelles étaient ces expériences personnelles du sacré ? Une ultime conversation au chevet de son lit d'hôpital quelques mois avant sa mort nous en avait brossé schématiquement l'histoire : depuis son enfance, qu'il s'amusait à appeler "magicienne », jusqu'à son initiation à une secte religieuse africaine. Bastide était, en effet, « Fils de Shango ».

Comment suppléer à cette auto-analyse projetée où ce chercheur aurait encore fait figure de pionnier ? On ne peut qu'essayer de lire en filigrane L'œuvre écrite qu'il émaille parfois de souvenirs personnels. Ainsi un récent article sur " Protestantisme et Médecine de Folk » témoigne de cette première période de sa vie : «Lorsque j'étais enfant, dans la région d'Anduze où je passais chaque année mes vacances, j'ai été nourri de ces histoires de sorcellerie, j'ai connu des gens ou des bêtes " enmasqués », on m'a retourné souvent la manche de ma chemise air passage de quelque vieille femme qui avait «le mauvais œil », car le mauvais oeil ne petit rien si on a un objet de sa toilette « à l'envers »... ${ }^{3}$. Ces « jeteurs de sort », ces " chères sorcières d'Anduze », dont il regrettait tant la disparition, ont par conséquent participé à créer chez ce jeune protestant cévenol une image très ambivalente du sacré. Très tôt fasciné par le monde magique, il le fut également par le monde onirique : « la séduction du monde des rêves date de mes

2 H.D., « Roger Bastide. Discours et parcours d'un dernier automne », Arch., 38, juillet-décembre 1974, pp. 4-8.

3 Revista de Etnografia (Porto), XV, 2, 1971, pp. 321-32. 
plus lointains souvenirs d'enfance » ${ }^{4}$. Le Rêveur, qu'il définira connue « l'intermédiaire privilégié entre le monde du surnaturel et celui de la nature ", appartient à ce monde des gouffres nocturnes, frontières du sacré dont Bastide reconnaît les dangers. "Dans ma quête passionnée des expériences mystiques - dit-il à Jacques Delpeyrou qui l'interroge, en 1965, pour le journal Combat - j'ai toujours en peur de devenir fou $» 5$.

Élevé à l'ombre du temple protestant, il n'est pourtant ni pratiquant ni lié à l'Église. De nombreuses critiques vis-à-vis de cet «Institué » jalonnent son oeuvre et peuvent expliquer ce refus d'appartenance. Comme, par exemple, celle-ci : «L'anthropologue découvre souvent à l'intérieur des Églises historiques au lieu de l'appréhension du sacré qu'il espérait y trouver, un ensemble de masques, d'apparences certes religieuses, mais qui couvrent de leurs mensonges des faits d'indifférence, voire de négation du pur religieux » ${ }^{6}$. C'est cette Église «figée » qu'il refuse, car elle administre le sacré en se « retournant contre le vécu pour l'emprisonner derrière les barreaux de ses dogmes ou dans sa liturgie bureaucratique ${ }^{7}$.

Sa passion pour le mysticisme qui préside sans doute à l'élaboration, en 1931, de son premier ouvrage, lui fait pressentir dès l'introduction que pour établir les lois de la vie mystique « seule la méthode comparative lui permettrait de multiplier les coups de sonde dans les milieux les plus divers, dans les pays les plus éloignés » ${ }^{8}$. On retrouve cette nécessité méthodologique du travail sur le terrain quatre années plus tard (1935) dans les Éléments de Sociologie Religieuse ${ }^{9}$. Bastide ajoute toutefois qu'« une sympathie intuitive ne doit pas man-

4 R.B., Le Rêve, la Transe et la Folie, Paris, Flammarion, 1972, p. 9.

5 J.D., Combat, lundi 27 déc. 1965, p. 2.

6 R.B., « Anthropologie Religieuse », Encyclopaedia Universalis, tome 1, p. 68.

7 R.B., « Le sacré sauvage » dans Le Besoin Religieux, Neuchâtel, La Baconnière, 1974, p. 134.

8 R.B., Problèmes de la vie mystique, Paris, A. Colin, 1931, p. 19.

9 R.B., Paris, A. Colin, 1935. 
quer au sociologue » pour appréhender le phénomène religieux. Ici encore une valorisation du vécu, nouvelle preuve de l'implication de l'homme dans l'oeuvre du chercheur. Cette "sympathie intuitive » sous-entend-elle une connaissance préalable de l'expérience mystique pour mieux l'appréhender, ou bien la nécessité d'accumuler ces expériences par la multiplication de terrains différents ? C'est une des multiples questions que pose son oeuvre.

Critiquant la sociologie livresque, ressentant peut-être ce besoin de mysticisme polythéiste qu'il valorisera dans ses derniers écrits à l'encontre du monothéisme chrétien, Bastide rencontre en 1938 des circonstances favorables qui lui permettent de concrétiser au Brésil ses « nécessités » de terrain.

C'est cependant cette période pré-brésilienne, qui nous semble capitale dans l'œuvre et la vie de Roger Bastide, car elle conditionne le choix de l'objet de recherche, l'appel vers la découverte de l'Autre. Lorsque la bibliographie exhaustive des articles de revues et journaux correspondant à ce premier volet bastidien sera disponible air chercheur, nous pensons qu'il y aurait là une mine de détails à glaner pour mieux saisir les motivations de cette quête mystique qui va conduire Bastide en Amérique Latine.

Le Brésil, cette " terre des contrastes », va donc être pendant près de seize ans le lieu privilégié de recherches sur l'entrecroisement des trois mondes africain, européen et indien. Seize années d'apprivoisement de l'Autre, qui ne représentent pas à proprement parler l'anthropologie religieuse de Roger Bastide, mais plutôt la période ethnographique, c'est-à-dire le recueil de données puis l'approche ethnologique qu'il définira lui-même comme «l'analyse du concret, le discours sur les dieux et les rites pour entrer en communication avec eux » ${ }^{10}$. Il y a chez l'anthropologue, selon lui, « comme une espèce de séduction

10 R.B., art. cit. dans Encyclopaedia Universalis, p. 65. 
des gouffres que constitue l'appel des civilisations primitives ${ }^{11}$. La séduction des gouffres pour Bastide c'est l'étude de la transe, du rêve, du mysticisme polythéiste... Ses élèves brésiliens, comme Maria Isaura Pereira de Queiroz, Florestan Fernandez, pourraient certainement nous apporter de très riches informations sur ces premières années de terrain, quelles furent ses méthodes de travail pour appréhender l'Autre, l'écouter et être accepté par lui jusqu'à partager son expérience existentielle du Sacré... Pour l'ethnologue (lue nous sommes, initiée aux vicissitudes de l'observation participante, l'«Alricanus sum, » que proclame Bastide dans l'Introduction des Religions Africaines du Brésil 12 résonne comme un hymne de joie et d'amour. Bien que blanc et de formation chrétienne, il fut en effet accepté par les membres noirs d'un candomblé yoruba et considéré, écrit-il, « comme frère en la foi avec les mêmes devoirs et les mêmes privilèges que les autres du même grade que moi " 13 . C'est d'ailleurs à ces soeurs et mères en la foi qu'il dédie l'un de ses derniers ouvrages, La Femme de Couleur en Amérique Latine, les remerciant d'avoir accueilli ce « fils blanc (...) cet Européen empêtré dans sa sauvagerie eartésienne »14. Nous retrouvons souvent dans l'oeuvre de Roger Bastide (et bien avant les « ethnocidaires » français) cette critique du moule occidental, véritable carcan idéologique qui empêche l'ethnologue de pouvoir comprendre « les infinies possibilités créatrices de l'homme, de les revivre, fût-ce maladroitement, empêtré qu'il est dans ses équipements européens » 15 .

L'ethnologie doit en effet « déchosifier les faits sociaux pour les humaniser elle doit cesser de coucher les civilisations entre les feuilles d'un herbier ou d'en cataloguer les manifestations comme des pièces

11 R.B., « Les cadres sociaux de l'anthropologie culturelle américaine », Cahiers Internationaux de Sociologie, 26, janv.-juin 1959, pp. 15-26.

12 Paris, P.U.F., 1960, p. 37.

13 Ibid.

14 Paris, Anthropos, 1974, p. 7.

15 R.B., «L'Ethnologie et le nouvel humanisme », Revue Philosophique, oct.déc. 1964, p. 447. 
d'un musée. Un mythe n'est pas une chose morte, une fleur désséchée, il vit et agit comme les songes d'un dormeur... »16. Contre la chosification de Durkheim, qui « n'avait d'autre but que de limiter les dégâts en exigeant de l'observateur qu'il ne juge pas des faits qu'il observe à travers ses préconceptions » 17 , il réhabilitera la notion de participation de Lévy-Bruhl, de même que, contre la pensée claire mais désincarnée parce (lue " vidée du religieux » de Lévi-Strauss, il valorisera la pensée confuse et obscure de Leenhardt 18 (18), parce que révélant ce jeu subtil de la vision du monde Canaque « entre le différent et l'identique, cet engluement de l'un dans l'autre et de l'autre dans l'un ». Mais pour atteindre cette connaissance de l'Autre, l'ethnologue doit, selon Bastide, se placer « au centre d'une culture » pour vivre et communier avec elle, "ne pas se contenter de faire parler les gens, mais apprendre à faire parler les choses et savoir les écouter » ${ }^{19}$. Ne pas seulement s'intéresser " aux moments chauds de la vie d'un peuple, c'est-à-dire aux grandes manifestations cérémonielles de leur vie religieuse », mais également " aux faits banals de l'existence quotidienne » 20 : Ainsi pourra-t-il faire parler le mythe, voir comment il s'inscrit sur le sol et s'incarne dans les objets quotidiens... car « tout est logos ou parole vivante ${ }^{21}$. Cette observation si particulière des choses nous la retrouvons dans ses carnets de notes très rapidement feuilletés, mais qui Prolifèrent de schémas et de remarques, dans son dernier compte rendu de mission au Brésil en septembre 1973 : « Je me suis aperçu dans le Nord-Est qu'au fur et à mesure que le nombre des candomblés se multiplie, les mythes qui sous-tendaient la liturgie étaient progressivement oubliées, ils ne subsistaient plus que sous

16 Ibid., p. 446.

17 R.B., « L'Expression de la prière chez les peuples sans écriture », La MaisonDieu, 109, 1972, p. 101.

18 R.B., « La Pensée obscure et confuse », Le Monde non-chrétien, 75-76, 1966, pp. 137-156.

19 R.B., « Mythologie », dans Ethnologie Générale, Paris, La Pléiade, 1968, p. 1075.

20 R.B., art. cit., 1972, p. 109.

21 R.B., « Mythologie », op. cit., p. 1075. 
forme de schémas musculaires ; j'ai donc mené une recherche parallèle avec observations et dessins sur le symbolisme gestuel (...) toute une série d'études sur le matériel recueilli est maintenant nécessaire, mon souci étant de théoriser à partir de l'empirique »...

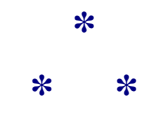

Si nous avons insisté dans ce deuxième volet brésilien sur l'approche méthodologique de Bastide, reconstituée d'ailleurs à partir d'écrits postérieurs, faute de l'avoir connu à cette époque, c'est parce que cette ethnologie "du dedans », du vécu, de la praxis, de la participation choisie par lui pour appréhender l'Autre et son mysticisme, est une des clefs de l'anthropologie bastidienne. Tout en gardant le secret des dieux auxquels il est initié, il saura en effet se servir de cette vision du dedans pour dépasser les concepts de "survivance », de "syncrétisme », avancés par d'autres auteurs pour expliquer les faits religieux afro-brésiliens, et s'apercevoir «qu'ils cachaient sous leur masque la permanence d'un système un et cohérent, qui était le système africain, en l'occurrence le système religieux yoruba » ${ }^{22}$. C'est ainsi que Bastide propose le «principe de coupure » pour caractériser les membres de ces candomblés qui, bien qu'intégrés dans le système économique et politique brésilien, pensaient, sentaient et vivaient en Africains. Comment cependant expliquer une telle fidélité à l'Afrique, alors que plusieurs générations s'étaient succédées depuis l'abolition de l'esclavage ? L'explication fonctionnaliste ne satisfaisant pas Bastide, il se rend très vite compte que cette fidélité ne peut finalement se comprendre qu'en «situant les Noirs dans la société des Blancs qui les frustrent comme individus, d'où la catharsis offerte par la transe mystique $" 23$. C'est ainsi que, parti de la transe - premier objet de recherche et motivation au départ -, Bastide est conduit à saisir le sens des

22 R.B., Texte inédit, 1972.

23 Ibid. 
afro-américanismes 24 en les replaçant dans l'ensemble des relations inter-raciales, comme mécanisme de défense des Noirs contre l'agression d'une société dominée et dirigée par les Blancs. et à substituer à l'analyse statique d'une culture une perspective dynamique qui le conduira du candomblé de la fin du XIXe siècle au spiritisme actuel de Umbanda.

Le retour en France et l'exploitation théorique des matériaux « empiriquement » recueillis permettent à Roger Bastide d'élaborer une nouvelle Anthropologie religieuse. Nous avons la chance d'avoir ce texte écrit pour l'Encyclopaedia Universalis, où il définit lui-même ce qu'il entend par cette science qu'il conçoit «indépendante » et non comme l'un des chapitres de l'Anthropologie sociale, \#situation dont elle a malheureusement trop longtemps souffert »: critique voilée mais certaine de l'école anglo-saxonne. L'Anthropologie religieuse doit essayer « de comprendre, par delà le chaos des faits religieux, l'homme en tant que manipulateur du sacré et constructeur de mondes symboliques » 25. S'intéressant plus à l'homme qu'à l'ethnie, les données de l'ethnologie ne lui servent qu'à mieux cerner, à travers les cultures qui sont son oeuvre, les lois générales de l'Homo religiosus. Par conséquent, l'Anthropologie religieuse devrait découvrir un système applicable aussi bien aux religions universalistes qu'aux religions des microsociétés. Ce qui frappe, en effet, particulièrement dans l'oeuvre écrite de ces seize dernières années, c'est ce souci constant de comparer les faits religieux africains ou afro-brésiliens avec des faits principalement chrétiens, afin de souligner non l'identique mais le différentiel. Que ce soit au niveau de la transe, de la prière, du mysticisme ou du messianisme, on retrouve cette même volonté de lutter contre l'ethnocentrisme occidental et de montrer la valeur et la richesse du pluralisme. «Le mysticisme africain, écrit Bastide, ne peut pas

24 Ceci le mènera notamment à l'étude des stéréotypes sur le nègre dans le folklore oral et dans la littérature érudite, puis sur la contre-agressivité par une analyse de contenu de la presse nègre.

25 R.B., « Anthropologie Religieuse », op. cit., p. 65, 68. 
être placé en dehors des autres formes de mysticisme hindou, musulman ou chrétien dans une espèce de compartiment inférieur que l'on appellera «mysticisme primitif » ou «folie collective », suivant que l'on est sociologue ou médecin. Il est comme les autres une forme authentique et passionnante, complexe et belle du mysticisme. Une des sources où les âmes altérées peuvent étancher leur soif et boire le ciel » 26 . Il refuse, en effet, d'opposer le religieux du primitif au rationnel chrétien, car il y a selon lui " plus de rationalité dans les cultures primitives - lesquelles valorisent la transe extatique, la magie agressive, le ritualisme -que dans la nôtre, où la raison s'est coupée de l'affectivité si radicalement qu'elle se laisse désormais conduire autant par des fantasmes que par les règles de la mathématique (...). Dans les sociétés primitives, au contraire, l'affectif est pris dans les réseaux du rationnel et doit obéir à ses lois » 27 . C'est une des raisons pour lesquelles Roger Bastide souligne si souvent le mérite de Leenhardt, qui a su démontrer « que la pensée mythique n'était pas un irrationalisme mais une " autre » rationalité que la nôtre ». On retrouve dans Le Rêve, la Transe et la Folie cette valorisation des primitifs " qui ont su établir une série de communications entre ces trois domaines, soit qu'une névrose ou un rêve soient considérés comme un appel des dieux (...) soit qu'au contraire le divin s'insinue dans les interstices des muscles, des viscères de l'homme dansant au rythme des tambours ", tandis que «nous, civilisés, avons rompu avec ces canaux de communication en naturalisant nos rêves, en enroulant nos malades mentaux dans des camisoles chimiques pour en faire des imitations de normaux, bref en rejetant le surnaturel pour le traduire en d'autres langues, plus satisfaisantes pour notre raison cartésienne, biologique et sociologique $» 28$.

26 R.B., « Réflexions sans titre autour des formes de la spiritualité africaine », Présence Africaine, XVII-XIX, 1958, pp. 9-16.

27 R.B., « Anthropologie Religieuse », op. cit., p. 66.

28 Op. cit., p. 5. 
En dehors de cette démystification constante de ce monopole occidental du rationnel que les soi-disant primitifs pratiquent « en domestiquant le sacré » - thème d'un des derniers et des plus prestigieux articles -, deux grands axes ordonnent la problématique de l'Anthropologie religieuse de Roger Bastide.

1) Considérer les religions comme des systèmes de symboles que l'on ne doit pas découper en morceaux (c'est-à-dire en rites, dogmes, mythes, etc.), mais saisir dans leur unité vivante comme une activité culturelle totale.

2) Envisager la religion comme tous les faits sociaux, c'est-à-dire comme quelque chose qui change tout en résistant et résiste tout en changeant.

Nous ne ferons que commenter très brièvement ces deux thèmes qui, en eux-mêmes, devraient faire l'objet de tout un livre pour bien saisir toute la portée de l'Anthropologie religieuse de Roger Bastide.

À propos du premier axe de recherche - considérer les religions comme des systèmes de symboles - là encore Bastide fait figure de pionnier. Et nous encouragerions vivement nos collègues américains à prendre connaissance de son oeuvre avant de dénoncer l'indigence de l'Anthropologie religieuse sur le plan théorique, connue le fit, par exemple, Clifford Geertz en 1966. Aucun progrès notable n'ayant été fait, selon ce dernier, depuis la première guerre mondiale, il constate que « si tant de travaux d'Anthropologie religieuse le laissent insatisfait, c'est qu'ils négligent l'analyse du système de significations incarnées dans les symboles qui constituent la religion proprement di- 
te " ${ }^{29}$. Nous renvoyons donc cet anthropologue à l'oeuvre de Roger Bastide, qui insiste depuis 1950 (près de quinze ans avant cette déclaration) sur l'importance des significations cachées, les modèles inconscients derrière les modèles conscients. La transe est en effet pour Bastide « un langage qui se décrypte selon un certain code qui a son vocabulaire, ses règles grammaticales et sa syntaxe " ${ }^{30}$. De même, le mythe est une parole « qui détermine les gestes de l'agriculteur, comme le plan de sa demeure, sa façon de manger, de faire l'amour et de mourir » 31 . Élaborer une grammaire de la transe, découvrir les structures syntaxiques du sacré sont les tâches urgentes de l'Anthropologie religieuse dont Roger Bastide nous a tracé les pistes par ses propres recherches.

La dimension diachronique des faits religieux, deuxième axe de sa problématique, permet de saisir la dynamique des religions, les métamorphoses des dieux et du sacré. Car pour lui le religieux se déplace, vit, évolue plus qu'il, ne disparaît. La multiplication des sectes due à l'urbanisation, la sécularisation des mythes qui deviennent celui du Héros, de la Vedette, sont autant de déplacements du religieux, car « la mort de Dieu n'est pas nécessairement la mort du sacré (...) la crise de l'Institué n'entraîne pas à sa suite une crise de l'Instituant, C'està-dire de l'effervescence des corps et des Cœurs ; c'est pourquoi (...) le sacré sauvage d'aujourd'hui se veut un sacré sauvage contre le sacré domestiqué des Églises » 32 . L'Anthropologie religieuse se doit donc d'étudier ces mécanismes de déplacement, comme ceux de remplacement, car la religion est, pour Roger Bastide, mémoire, mais aussi création pour répondre aux défis lancés par la société. C'est ainsi qu'il différenciera « les religions en conserve » comme celle du candomblé, des « religions vivantes » comme le vaudou haïtien ou le spiritisme de

29 C.G., Anthropological Approaches to the Study of Religion, Londres, ASA Monographs, 1966.

30 Le Rêve, la Transe et la Folie, op. cit, p. 96.

31 «Anthropologie Religieuse », op. cit., p. 66.

32 «Le Sacré sauvage », op. cit., p. 137. 
Umbanda ${ }^{33}$. Le messianisme est aussi une forme de création religieuse, en ce sens qu'il est utilisé par l'indigène comme technique pour recouvrer un sentiment de dignité que le mépris de l'Européen avait un moment dégradé 34 .

Voilà les premières réflexions, les premières questions qui s'imposent au chercheur lorsqu'il tente de saisir l'Anthropologie religieuse de Roger Bastide qui va inspirer, dans les années à venir, de nombreuses recherches. Nous sommes en effet convaincue avec J. Le Goff 35 que l'audience et la stature de ce pionnier ne cesseront de grandir.

Roger Bastide n'a pas, à l'instar de ses collègues français contemporains, créé une école ou forgé une méthode qui est "religieusement » et inlassablement appliquée par des dizaines d'élèves. Il nous en donnait, dans une lettre récente, la principale raison : "Comme Gide, je hais les disciples... ». À nous maintenant de savoir lire son œuvre qui fourmille de conseils, d'idées nouvelles, de pistes à suivre.

Bien qu'ennemi d'une certaine anthropologie appliquée, Roger Bastide a su, et particulièrement dans l'oeuvre des dix dernières années, mettre ses connaissances sur le pluralisme religieux et les richesses d'un " autre » sacré au service d'institutions comme celle des Missions Protestantes, dont il condamne sévèrement certaines oeuvres : « ce sont nos églises métropolitaines qui ont besoin maintenant pour revivre de missionnaires noirs ou jaunes - non bien entendu de missionnaires qui seraient ces copies de leurs anciens maîtres, mais qui nous révéleraient la communion, la participation, la symbolique

33 Les Amériques Noires, Paris, Payot, 1967, 236 p.

34 R.B., «Le Millénarisme comme stratégie de la recherche d'une nouvelle identité, et dignité », dans Le Sacré Sauvage (réunion posthume par H. Desroche de textes sur le sacré) Paris, Payot, 1975, 236 p.

35 Hommage funèbre. 
d'un christianisme vraiment asiatique ou enraciné dans la négritude » 36. Ou bien encore, dans Le Prochain et le Lointain : "Nous avons mis notre Dieu sous vitrine avec des boules de naphtaline pour qu'il se conserve tel quel, séparé du monde par une vitre ou une porte de bois ; il faut briser la vitre ou la porte pour faire revenir le divin sur terre... » 37 .

Ces appels aux dieux sont, selon nous, une réponse aux questions que se posait le Bastide des années 30. En d'autres termes, pour comprendre "Le Sacré Sauvage » il faut revivre l'itinéraire intellectuel et religieux de ce protestant cévenol. Les derniers écrits faisant écho aux premières questions, - «Les problèmes de la Vie Mystique » - doivent nous fournir le fil d'Ariane de son Anthropologie religieuse.

Françoise MORIN

Université de Toulouse-Le Mirail

36 R.B., « Sociologie des Missions protestantes », Arch., 8, 1959, p. 56.

37 Paris, Cujas, 1970, p. 12. 